\title{
Des manèges aux tipis. « Équitation éthologique » et mythes indiens
}

Jean-Pierre Digard

\section{(2) OpenEdition}

\section{Journals}

Édition électronique

URL : https://journals.openedition.org/tc/1139

DOI : $10.4000 /$ tc. 1139

ISSN : 1952-420X

Éditeur

Éditions de l'EHESS

\section{Édition imprimée}

Date de publication : 1 décembre 2004

ISSN : 0248-6016

\section{Référence électronique}

Jean-Pierre Digard, «Des manèges aux tipis. «Équitation éthologique » et mythes indiens »,

Techniques \& Culture [En ligne], 43-44 | 2004, mis en ligne le 15 avril 2007, consulté le 29 septembre 2022. URL : http://journals.openedition.org/tc/1139; DOI : https://doi.org/10.4000/tc. 1139

Ce document a été généré automatiquement le 29 septembre 2022

Tous droits réservés 


\title{
Des manèges aux tipis. «Équitation éthologique » et mythes indiens
}

\author{
Jean-Pierre Digard
}

1 Les activités humaines liées au cheval viennent de connaître, en Europe, dans la deuxième moitié du XXe siècle, une révolution comparable, par ses répercussions, à la généralisation de la cavalerie il y a 3000 ans ou à l'élaboration de la selle à arçon il y a 2000 ans en Eurasie. Mais les derniers changements présentent, tant dans leurs formes que dans leurs déterminants, des aspects profondément originaux, avec parfois des prolongements interculturels inattendus ${ }^{1}$.

La révolution équestre du XXe siècle

2 En quoi a consisté cette révolution? Au début du XXe siècle, même si la cavalerie ne jouait plus dans les batailles un rôle décisif, l'équitation restait majoritairement militaire. Ou si elle était civile, elle demeurait presque exclusivement masculine. (Comme le montre Catherine Tourre-Malen dans ce volume, les femmes à cheval représentaient une minorité et montaient selon une technique particulière, dite «en amazone ", qui s'avérait relativement handicapante.) Civil ou militaire, le monde équestre était tout entier dominé par la figure de l'«homme de cheval» héritée du XIXe siècle, époque par excellence de la prolifération et de l'omniprésence des chevaux (Digard 2004 : chap. VII). À doses homéopathiques à partir des années 1930 et après la Deuxième Guerre, puis massivement à partir des années 1960, de nouveaux facteurs économiques, sociaux, culturels- sont venus bouleverser ce paysage équestre.

3 Premier indicateur de ces changements: la démographie équine. En France par exemple, le cheptel chevalin déclaré, qui s'était maintenu à quelque trois millions de têtes jusque vers 1935, décline à partir de cette date pour tomber, au milieu des années 1980, à 300000 têtes avec un taux de pénétration d'un cheval pour 200 habitants. Les chevaux de trait, qui constituaient la plus grande partie du cheptel, sont les premières victimes de cet effondrement: on en compte 2,5 millions avant la guerre contre une trentaine de milliers à la fin du XXe siècle. La situation des chevaux de selle montre, quant à elle, une double évolution : leur nombre absolu baisse de plus de la moitié entre 
1930 et 1970 mais, du fait de l'importante diminution des traits, leur part de l'effectif équin progresse constamment : 17 \% en 1935, 38 \% en 1970, 64 \% en 1978, 91 \% en 1995.

4 Pour le cheval de selle -on n'insistera jamais assez sur la coupure sociale et culturelle qui sépare le monde du trait et le monde de la selle (Lizet 1988 et 1996)-, l'élément déterminant a été le glissement du centre de gravité de l'univers équestre du domaine militaire vers le domaine civil. Grande aînée des bastions traditionnels du cheval, l'armée, par étapes successives, a fini par renoncer presque entièrement à son auxiliaire emblématique. Il aura suffi d'une génération -vingt-cinq ans- pour que l'équitation passe presque entièrement des quartiers de cavalerie aux clubs équestres, et les chevaux de selle de la sphère de l'utilitaire à celle des loisirs. En France toujours, tandis que le nombre total des chevaux, de trois millions en 1935, décroissait régulièrement pour se stabiliser à partir de 1970 autour de 400000 chevaux, le nombre des cavaliers réguliers progressait, lui, d'une trentaine de milliers au lendemain de la dernière guerre à environ 620000 en 2001, dont 432500 licenciés à la Fédération française d'équitation.

5 Outre leur augmentation numérique, les « usagers » du cheval ne sont pas non plus les mêmes sur le plan sociologique. Alors que l'« homme de cheval» issu du XIXe siècle était un officier et/ou un aristocrate -un homme en tout cas-, les statistiques de la fin du XXe siècle montrent que les «nouveaux cavaliers » sont en majorité des femmes (à plus de $70 \%$ ), des jeunes ( $67 \%$ des licenciés ont moins de 25 ans, $58 \%$ moins de 18 ans) -tendance accentuée par un fort taux d'abandon à partir d'un certain niveau d'apprentissage (Chevalier 1990)-, des citadins, membres des classes moyennes (cadres, enseignants, professions libérales, commerçants aisés) (Chevalier \& Dussart 2002 : 465-466). Le champ des activités équestres a ainsi été investi par des catégories sociales qui s'en trouvaient auparavant exclues: c'est ce qu'on a appelé (improprement) la « démocratisation » de l'équitation (il serait plus exact de parler de sa « massification »).

Les changements survenus sont enfin et surtout culturels: la culture de l'homme de cheval, fondée sur l'utilisation polyvalente et intensive mais respectueuse de l'animal, qui a régné sans partage dans les milieux équestres jusque dans les années 1960, est aujourd'hui en passe d'être balayée par la nouvelle culture équestre -baroque, hédoniste et sentimentale- des "nouveaux cavaliers». Baroque en ce sens qu'elle se nourrit d'une diversification sans précédent des pratiques équestres : aux trois grandes disciplines classiques -dressage, concours de saut d'obstacles (CSO) et concours complet d'équitation comportant un cross (CCE) - s'ajoutent ou se substituent des disciplines nouvelles ou autrefois méprisées comme le horseball, les pony-games, l'équitation américaine ( «western »), la doma vaquera espagnole et autres équitations "exotiques", auxquelles l'équitation nouvelle emprunte des éléments disparates. Hédoniste parce que la pratique équestre majoritaire est désormais orientée moins vers l'effort et la compétition sportive que vers la recherche de sociabilité, de " nature ", de détente et de plaisir sans contrainte. Sentimentale, enfin, du fait de l'accession du cheval à un nouveau statut culturel. Dans l'ancienne culture en effet, le cheval était apprécié à hauteur des services qu'il rendait; l'« homme de cheval » mettait un point d'honneur à respecter l'animal, en vertu du principe de bon sens selon lequel «qui veut aller loin ménage sa monture». Aujourd'hui, dans la hiérarchie occidentale des animaux domestiques, le cheval occupe une position d'« animal intermédiaire »: il ne fait plus partie des animaux de rente, à fonction strictement utilitaire, et son statut tend de plus 
en plus vers celui de l'animal de compagnie (Digard 1999 : chap. III). D'ores et déjà, Européens de l'Ouest (Milliet et Digard 1989) et Américains du Nord (Berger 1989) placent le cheval en troisième position, juste derrière le chien et le chat, dans l'ordre de leurs faveurs. La revue Cheval Magazine a d'ailleurs explicitement réclamé, à l'occasion de la préparation de la loi française du 6 janvier 1999 relative à la protection des animaux, que le cheval soit, au même titre que le chat et le chien, légalement admis dans la catégorie des " animaux de compagnie $»^{2}$.

7 Ainsi promu, le cheval devient l'objet de tous les soins, de toutes les attentions, de toutes les compassions. La moindre "brutalité » ou " cruauté » provoque l'indignation vertueuse de la gent cavalière : elle, traditionnellement si soucieuse de convenances et de retenue, n'hésite plus aujourd'hui à huer tel cavalier d'obstacle sanctionnant de la cravache une faute de sa monture ou tel cascadeur dont la prestation est jugée trop dangereuse ou brutale pour les chevaux. Depuis peu, dans les compétitions officielles, les sanctions sont strictement réglementées : en CSO, le nombre quotidien de parcours est limité et les chutes sont éliminatoires; en CCE, des parcours routiers et des steeples sont supprimés, etc. D'une façon générale, des critiques de plus en plus sévères sont systématiquement portées par certains mouvements protectionnistes, et complaisamment relayées et amplifiées par la presse équestre populaire, à l'encontre de pratiques comme la caudectomie, le marquage au fer (interdit dans plusieurs pays de l'Union européenne), le «barrage » des chevaux d'obstacle ${ }^{3}$, le surharnachement des trotteurs, la longueur et le rythme des épreuves d'endurance, le danger (pour les chevaux) des obstacles fixes sur les parcours de cross (des associations américaines ont activement milité pour faire interdire l'épreuve de cross des Jeux Olympiques d'Atlanta en 1996); demain, peut-être, le dressage, le mors, la selle seront visés à leur tour... La «monte en mains $»^{4}$ est dénoncée comme un "viol de la jument ${ }^{5}$. Les conditions « inhumaines» (sic) de transport des chevaux de boucherie suscitent périodiquement de violentes campagnes de dénonciation. L'hippophagie, du reste en stagnation, répugne aux "nouveaux cavaliers», de sorte que, depuis 1990 environ, les centres équestres, de peur de perdre leur clientèle, n'osent plus envoyer (du moins directement) à la boucherie leurs chevaux de réforme. Les « maisons de retraite " pour chevaux et les « refuges pour équidés martyrs » se multiplient, et l'idée de cimetières pour chevaux commence à poindre...

On sait que ce qui distingue l'animal de compagnie des autres animaux domestiques, c'est de ne servir à rien d'autre qu'à la compagnie de son maître. En Europe, le chien à partir de la Renaissance, le chat à partir du XVIIIe siècle ont vu leurs fonctions économiques s'effacer peu à peu au profit des seules fonctions affectives et cathartiques. De tels changements de statut s'observent aussi, bien qu'à un degré moindre, dans d'autres cultures : chez les Indiens et les Inuit du Canada, par exemple, il arrive que des couples sans enfant adoptent des chiens; ceux-ci cessent alors de travailler et d'être durement traités comme le sont les chiens de traîneau (Digard 1990 : 196-200, 234-235 et chap. XI). En Occident, c'est dans la seconde moitié du XXe siècle que le statut du cheval connaît semblable évolution : jamais il n'a été aussi protégé ni autant aimé que depuis qu'il ne "sert " plus à la guerre, aux champs ou sur les routes. Aujourd'hui, le cheval de selle est de moins en moins perçu en tant que monture; des enquêtes en cours dans des centres équestres, venant amplifier les résultats de sondages plus anciens, indiquent qu'un nombre significatif de cavaliers de base se montrent, au fond, plus intéressés par la fréquentation des chevaux que par la pratique de l'équitation. Un nombre croissant d'amateurs -femmes pour la plupart, ce qui n'est 
sans doute pas un hasard (Digard 1995 et Tourre-Malen 2003) - acquièrent des chevaux à seule fin de... ne pas les utiliser. En témoignent, par exemple, la prolifération dans le sud de la France des « chevaux potagers ", gardés à ne rien faire dans un coin de pré, de jardin ou de garage, ou encore la progression lente mais certaine des "chevaux d'appartement ", falabellas et autres « horse toys » de $45 \mathrm{~cm}$ au garrot, dont on peut déjà se procurer les couches (!) dans le commerce.

La tocade de l'« équitation éthologique »

9 C'est dans ce contexte que sont apparues les « équitations alternatives » et, au premier rang d'entre elles, l'équitation auto-proclamée « éthologique », suscitant actuellement un engouement qui mérite examen.

10 L'approche du cheval désignée aujourd'hui comme "équitation éthologique " a été élaborée aux États-Unis en réaction contre les méthodes traditionnellement employées par les cow-boys pour dresser les chevaux (to break horses), méthodes brutales car expéditives par nécessité économique. Au contraire, les «nouveaux maîtres » principalement Tom Dorrance (né en 1910), puis Ray Hunt (1929), Monty Roberts (1935), John Lyons (1947) et Pat Parelli (1954), le plus célèbre aujourd'hui- prétendent "persuader le cheval plutôt que le soumettre", en s'efforçant de "se mettre à sa place » pour « penser comme lui » ${ }^{6}$.

11 Un film, réalisé et interprété par Robert Redford, tiré d'un roman de Nicholas Evans, L'homme qui murmurait à l'oreille des chevaux (1996), lui-même inspiré de l'autobiographie de Monty Roberts, L'homme qui sait parler aux chevaux. Histoire de ma vie (1995), ont beaucoup contribué à la renommée de ces «nouveaux maîtres", appelés aussi " chuchoteurs ». Les méthodes de marketing et de " communication » à l'américaine, déployées notamment par un Pat Parelli à partir de son relais français du Haras de la Cense dans les Yvelines, ont fait le reste. Toujours est-il que le succès populaire remporté par les « chuchoteurs » auprès des «nouveaux cavaliers » est aujourd'hui tel que la Fédération française d'équitation elle-même, visiblement soucieuse de ne laisser échapper aucun licencié potentiel, cherche à intégrer ce courant en créant des diplômes fédéraux dans une activité promue du même coup au rang de discipline équestre.

Mais ce serait passer à côté de l'essentiel que d'attribuer ce succès aux seules « vertus » du marketing. Une large part de l'activité des «nouveaux maitres » consiste en clinics destinés aux chevaux difficiles, rétifs ou affublés de comportements gênants (la quatrième de couverture de L'Homme qui sait parler aux chevaux précise que «Monty Roberts accomplit des miracles. Cet homme capable de dresser les chevaux les plus indomptables ou de guérir les plus incurables, refuse pourtant d'être pris pour un sorcier »). Or, pour des raisons évidentes, les cavaliers ont toujours été -sont de plus en plus- friands de trucs et de recettes propres à ramener sur le droit chemin les chevaux récalcitrants. Plus en profondeur, l'équitation « éthologique » apparaît -à tort ou à raison, de manière justifiée ou non - comme une synthèse, plus ou moins habile ou réussie, d'ingrédients au goût du jour, comme porteuse d'attitudes et de valeurs qui sont incontestablement dans l'air du temps.

13 Le premier de ces éléments est l'étiquette « éthologique » qu'affiche ce courant. Cette référence au nom d'une discipline scientifique apparaît manifestement abusive dès lors que le corpus de connaissances et de pratiques des "nouveaux maîtres » n'est rien d'autre qu'empirique, le plus souvent un habillage remis au goût du jour de connaissances et de pratiques qui sont familières à la plupart des praticiens du cheval. 
À ce compte-là, toutes les équitations sont nécessairement "éthologiques " (au sens commun) et celle des «chuchoteurs " ne l'est pas plus que les autres. À ce propos, la Société française pour l'étude du comportement animal a justement rappelé dans un récent communiqué de presse que :

«Le statut d'éthologiste requiert une formation et des connaissances spécifiques sur le comportement animal. [...] il est abusif de parler d'équitation éthologique, de stages d'éthologie ou de formation à l'éthologie pour désigner des stages d'équitation encadrés par des dresseurs ou des enseignants diplômés d'équitation, ceci ne préjugeant en rien de leurs qualifications dans leur domaine de compétence propre. [...] il serait malheureux que l'éthologie ne soit mentionnée que pour servir de caution scientifique à des discours qui ne reposent sur aucune connaissance éthologique. ${ }^{7}$

Il est évident, en effet, que par cette référence à l'« éthologie ", les "chuchoteurs " veulent surtout donner à croire que le cheval serait ici plus qu'ailleurs le centre de l'activité équestre, en une sorte de version «scientifique » de la vision de tel auteur d'une revue équestre populaire évoquant l'image du centaure: c'est "l'homme qui prolonge son cheval » (Bigo $1986: 28$ ).

De cette première illusion, les « nouveaux maîtres " glissent tout naturellement vers la supercherie qui consiste à faire croire au chaland, par une subtile mise en scène des apparences de la non-violence physique, que les méthodes des "nouveaux maitres » seraient fondées sur l'absence de domination du cheval mais aussi sur sa persuasion. Cette mise en scène n'abuse que les ignorants car, dans la réalité, il n'est guère laissé de choix au cheval! Très éclairant est, à cet égard, l'exemple de l'« équitation à mains nues » de Pat Parelli : l'insistance mise sur l'absence spectaculaire de rênes et de mors a pour effet de détourner l'attention des longs éperons à molette qui arment ses bottes... Ce procédé de déplacement est bien connu des prestidigitateurs, comme est connue des (fins) cavaliers la technique "main sans jambes, jambes sans main » mise au point et publiée par l'écuyer François Baucher en... 1864 ! (Bacharach 1986 : 108).

Troisième aspect marquant, l'essentiel du travail des «chuchoteurs » s'effectue, non pas en selle, mais à pied. Leurs manipulations du cheval s'apparentent davantage aux concours d'agility canins qu'aux sports équestres et introduisent le cheval dans un processus sournois de canisation. Flagrant sous-emploi du cheval, l'« équitation éthologique " s'inscrit, de facto, dans le processus actuel de régression des usages du cheval et de sa transformation en animal de compagnie.

Mythes d'Indiens ou sur les Indiens?

Un dernier aspect de l'« équitation éthologique " ne manque pas d'intriguer. C'est la référence, plus ou moins constante, plus ou moins affirmée, à un legs équestre des Indiens d'Amérique du Nord. Certains «nouveaux maîtres » ne se cachent pas d'avoir puisé à cette source les principes fondamentaux de leur méthode : " harmonie » entre le cheval et l'homme pour Ray Hunt, «non-violence » dans leurs rapports pour Monty Roberts, le «sorcier $»^{8}$. Dans le même temps, la mode de l'« équitation éthologique » a suscité une curiosité et un engouement nouveaux pour les Indiens. En France, la journaliste équestre Maria Franchini a publié, dans la collection «L'Équitation autrement » (Zulma), une vaste et fantaisiste compilation sur « la civilisation indienne » et le cheval (Franchini 2001 : 15). Récemment, dans un «dossier» significativement intitulé « Les Indiens : une culture très tendance », la revue Cheval Loisirs ${ }^{9}$ a interrogé la même journaliste; à une question de la revue ainsi formulée : "J'aimerais croire qu'ils 
[les Indiens] sont à l'origine des techniques dites "éthologiques" pratiquées par les chuchoteurs d'aujourd'hui. Qu'en pensez-vous? », Maria Franchini répond :

«J'en suis persuadée à $100 \%$. Les Indiens avaient compris que les chevaux communiquent par le langage du corps. Ils utilisaient aussi beaucoup la voix. Peuples nomades, ils n'avaient ni écuries, ni récoltes de foin. Ils accueillaient leurs montures sous leur propre tente. Ils n'étaient pas "mieux" que nous, mais leur mode de vie les obligeait à tout savoir pour que leurs chevaux survivent. »

On voit donc ainsi s'échafauder une vulgate qui pourrait se réduire aux trois propositions suivantes : 1) il existe une seule civilisation indienne, forcément équestre (même quand cela n'est pas précisé, c'est toujours d'Indiens d'Amérique du Nord qu'il s'agit); 2) cette civilisation indienne est porteuse des valeurs d'harmonie et de nonviolence dans les rapports homme-cheval; 3) les «chuchoteurs » modernes sont les héritiers de la civilisation équestre indienne.

19 Il n'y a pas lieu de s'attarder sur le premier postulat, sinon pour souligner son absurdité. Pour s'en tenir au cheval, on ne peut que rappeler que cet animal, introduit par les Espagnols en Amérique du Sud et dans l'actuel Mexique, est parvenu aux différentes sociétés indigènes à des dates (échelonnées entre le début du XVIe et la fin du XVIIIe siècle), selon des modalités (par imitation des Conquérants ou en réaction contre eux), sous des formes (domestiques ou "marrons", avec ou sans «mode d'emploi », par transfert ou non au cheval des techniques et de la terminologie du chien $^{10}$ ) et pour des usages (fourniture de viande, cuir et graisse, auxiliaire de chasse ou d'élevage, moyen de transport, arme de guerre) extrêmement variés ${ }^{11}$.

De même pour le deuxième postulat, il est possible de glaner dans les sources crédibles autant d'indices du respect que les Indiens (ou certains d'entre eux) portaient à leurs chevaux que de preuves qu'ils (ou que certains autres) les traitaient «comme des bicyclettes", ainsi que le déplorait l'anthropologue Ralph Linton à propos des Comanches (1936: 428-429), ou encore que de faits pouvant donner lieu à des interprétations divergentes: par exemple, les Sioux des Plaines dressaient-ils incomplètement leurs chevaux par compassion ou pour les rendre plus difficiles à voler? Cette dernière difficulté tient à la coexistence, dans de nombreuses sociétés d'« Indiens cavaliers ", de pratiques d'élevage et d'une idéologie liée à la chasse, selon laquelle "le gibier est soit un alter ego en position d'extériorité absolue quand il est chassé, soit trop identique à soi pour être mangé lorsqu'il est apprivoisé » (Descola 1994 : 340; Desveaux $2001: 163-170$ ), de sorte que si les chevaux y sont souvent traités comme des humains, les humains peuvent tout aussi bien y être traités comme des chevaux, selon une classique formule d'homologie structurale. Loin d'un modèle d'harmonie et de non-violence entre l'homme et ses frères animaux, comme le voudrait la lecture "écologiste" selon laquelle les Indiens voient dans les animaux des «personnes non humaines » (Feit 2000), il s'agit au contraire, fondamentalement, d'une « idéologie prédatrice qui voit dans la capture chez autrui de personnes, de substances et d'identités une condition nécessaire à la reproduction du groupe local » (Descola 1994 : 339).

21 La réalité est donc fort éloignée des méthodes «naturelles» inspirées d'un idéal amérindien d'harmonie retrouvée entre l'homme et ses frères animaux, dont troisième postulat- les « chuchoteurs " seraient les héritiers spirituels. Pourquoi donc pareille distorsion? Plusieurs explications peuvent être ici sollicitées. Il faut se souvenir, en premier lieu, que les méthodes des «chuchoteurs » sont nées aux ÉtatsUnis où elles se posaient en « alternative » à la violence des techniques de débourrage 
(breaking) des chevaux par les cow-boys, ennemis jurés des Indiens. À l'appui de cette interprétation, on peut signaler le fossé qui sépare la clientèle de l'«équitation éthologique » de celle de l'« équitation américaine » ("western »). Plus largement, on peut voir, en second lieu, dans ce nouvel avatar du mythe du "bon sauvage » une manifestation de la moderne "nostalgie de l'état sauvage » (Belorgey $2000: 29-50$ ) avec son paradoxe qui consiste à ériger le "sauvage » en modèle pour le « civilisé ». Dans ce discours, convergent curieusement des arguments de marketing -rénovation de recettes banales par un vernis d'exotisme- et des thèmes chers au néo-paganisme d'extrême-droite -rejet du matérialisme occidental, fascination pour les croyances et les panthéons païens (souvenons-nous, entre autres, des colères droitières de l'écrivain voyageur Jean Raspail fulminant de n'avoir point trouvé de « vrais » Indiens, nobles et pur-sang, dans les réserves des États-Unis) (Raspail 1975).

Un précurseur des « chuchoteurs»?

Et si les «nouveaux maîtres» avaient un précurseur? C'est ce que prétend l'éditeur Jean-Louis Gouraud dans sa présentation de la réédition de l'ouvrage de John S. Rarey, L'art de dompter les chevaux, publié pour la première fois en anglais (The Art of Taming Horses) en 1858. Personnage énigmatique, et qui fit couler beaucoup d'encre, que ce Rarey. Se produisant au cirque à Paris fin 1857-début 1858, il fit sensation en venant à bout, par de mystérieuses méthodes fondées sur l'observation du comportement animal, d'équidés réputés indomptables (Digard $2002: 28$ ). Outre qu'il fournit à Nicolas Leskov le personnage de son « Vagabond enchanté » (Leskov $1967: 188$ et suivantes), il suscita surtout les analyses et les commentaires enthousiastes ou sceptiques des écrivains et chroniqueurs équestres de l'époque.

Les uns le désignent comme un "rough rider» (Saurel 1990: 421) ou comme "le dompteur féroce» (Leskov 1873: passim) ou encore décrivent ses procédés -par exemple : mettre un cheval difficile sur trois membres en ligaturant l'un des antérieurs (Rarey 1858 : 96) - comme impitoyables et sans autre issue pour le cheval que l'entière soumission (Vergnaud 1860 : 289; Le Bon 1990 : 119) : «Pour obtenir l'obéissance [du cheval], il faut s'en faire craindre ", écrivait Rarey (1858:63).

Les autres auteurs s'efforcent de tempérer plus ou moins cette image implacable :

«C'est à tort, à mon avis qu'on l'a [Rarey] accusé de brutalité ou de cruauté. Je l'ai vu opérer, et il montrait toujours une fermeté absolue, mais sans aucune violence. » (Mennessier de la Lance 1915, vol. II : 377)

Un cavalier contemporain se montre encore plus indulgent :

« La méthode Rarey [...] soumet complètement, sans douleur et sans faire appel à la notion de punition [...]. La méthode Rarey permet une approche [...] douce de l'animal agressif ou apeuré et engage le processus de confiance dans le meneur. » (Brager 1995 : 70-71)

Enfin, certains auteurs ne manquèrent pas de se faire l'écho de l'affairisme de Rarey et des démêlés qu'il lui attira. En effet, raconte François-Paul de Guaita, traducteur et préfacier de l'édition en français de la méthode de Rarey en 1858 :

«Il faisait un secret de sa méthode, mais moyennant une somme une fois payée de deux cent cinquante francs, il se chargeait de l'enseigner aux amateurs de chevaux, qui devaient promettre de garder le précieux secret, et s'engager, au cas où ils ne tiendraient pas parole, à payer à l'habile dompteur une amende de douze mille cinq cents francs. " (in Rarey 1858 : VI) 
Rarey commençait à amasser ainsi une solide fortune lorsqu'un incident singulier se produisit :

«À l'une des séances du professeur, à Londres, un de ses élèves, M. Leslie, montra une brochure écrite en 1856 par M. Rarey sur l'art de dompter les chevaux sauvages, et se plaignit fort amèrement qu'on lui eût fait payer deux cent cinquante francs un secret que chacun pouvait acheter pour six pence. $»^{12}$

L'affaire fit grand bruit dans les milieux équestres et dans la presse des deux côtés de la Manche mais n'affecta guère, semble-t-il, la renommée de la méthode de Rarey.

Malgré le siècle et demi qui les sépare, l'expérience du célèbre « dompteur » et celle des « chuchoteurs » modernes présentent en gros les mêmes ingrédients : procédés pour venir à bout de chevaux à problèmes, argumentation "éthologique", débat sur le traitement des chevaux, succès populaire et commercial... Seule exception notable : la référence à une culture équestre indienne aussi artificielle qu'imprécise. En effet, Rarey a longuement vécu au contact des Indiens dans son Ohio natal (Saurel 1990 : 421; Evans 1996: 104) mais, à la différence des «nouveaux maîtres» de l'« équitation éthologique ", il n'en fait jamais état dans sa méthode. Au contraire, les « chuchoteurs " l'utilisent généreusement pour relever leur sauce. La référence indienne, largement mythique, explique au moins autant que la référence éthologique, le succès des «nouveaux maitres » auprès d'un public occidental désormais coupé de toute culture équestre vivante.

\section{BIBLIOGRAPHIE}

Bacharach, René

1986. Réponses équestres. Lausanne : Caracole.

Belorgey, Jean-Michel

2000. Transfuges. Voyages, ruptures et métamorphoses : des Occidentaux en quête d'autres mondes. Paris : Autrement (« Mémoires »).

Berger, Joël

1989. « The horse: Noble steed or wild menace?», pp. 101-112, in R. J. Hoage (ed.), Perceptions of Animals in American Culture. Washington D. C. : Smithsonian Institution Press.

Bigo, Stéphane

1986. «Le cheval, mythe ou réalité ? ", Cheval Magazine 186 : 26-28.

Brager, Émile

1995. Techniques du voyage à cheval. Paris : Nathan.

Chevalier, Vérène

1990. «Les pratiquants de l'équitation : une population et ses mouvements », Population 45, 3 :

661-666. 
Chevalier, Vérène \& Brigitte Dussart

2002. «De l'amateur au professionnel : le cas des pratiquants de l'équitation », L'Année Sociologique $52,2: 459-476$.

Descola, Philippe

1994. « Pourquoi les Indiens d'Amazonie n'ont-ils pas domestiqué le pécari ? Généalogie des

objets en anthropologie de l'objectivation », pp. 329-344, in B. Latour et P. Lemonnier (eds.), De la préhistoire aux missiles balistiques. L'intelligence sociale des techniques. Paris : La Découverte/

Fondation « Les Treilles ».

Desveaux, Emmanuel

2001. Quadratura americana. Essai d'anthropologie lévi-straussienne. Genève : Georg.

Digard, Jean-Pierre

1990. L'Homme et les animaux domestiques. Anthropologie d'une passion. Paris : Fayard (« Le Temps des Sciences »).

1992. « Un aspect méconnu de l'histoire de l'Amérique : la domestication des animaux », L'Homme 122-124 : 253-270.

1995. "Cheval, mon amour. Sports équestres et sensibilités "animalitaires" en France », Terrain $25: 49-60$.

1999. Les Français et leurs animaux. Paris : Fayard.

2002. Le Cheval, force de l'homme. Paris : Gallimard (" Découvertes »).

2004 Une histoire du cheval. Art, techniques, société. Arles : Actes Sud.

s/presse. « Du travail au plaisir, du respect à la compassion : fonctions et statuts du cheval de selle dans la France du XXe siècle ", in Daniel Roche (ed.) Le Cheval et les loisirs (XVIe-XXe siècle). Paris : Association pour l'académie d'art équestre de Versailles.

Digard, Jean-Pierre, Laurence Ould Ferhat \& Catherine Tourre-Malen

1998. «Cheval et sciences sociales », L'Équitation (Saumur) 14 :13-19.

Evans, Nicholas

1996. L'Homme qui murmurait à l'oreille des chevaux. Paris : Albin Michel.

Feit, Harvey A.

2000. «Les animaux comme partenaires de chasse. Réciprocité chez les Cris de la baie James », Terrain $34: 123-142$.

Franchini, Maria

2001. Les Indiens d'Amérique et le cheval. Paris : Zulma.

Hunt, Ray

2002. L'Homme et le cheval en harmonie. Arles: Actes Sud (1ère édition 1978).

Kiley-Worthington, Marthe

1999. Le Comportement des chevaux. Pour une amélioration du bien-être des chevaux, de leurs conditions d'élevage et d'entraînement. Paris : Zulma (1ère édition 1989).

Le Bon, Gustave

1990. L'Équitation actuelle et ses principes. Paris : Jean-Michel Place (1ère édition 1892).

Leskov, Nicolas

1967. « Le Vagabond enchanté », pp. 177-359, in Lady Macbeth au village, L'Ange scellé, Le Vagabond enchanté, Le Chasse-Diable. Paris : Gallimard («Folio ») (1ère édition 1873).

Linton, Ralph

1936. The Study of Man : An Introduction. New York : Appleton-Century-Crofts. 
Lizet, Bernadette

1988. « La liaison dangereuse. Enjeux sociaux du clivage entre les chevaux de selle et les chevaux de trait ", pp. 131-142, in J.-P. D. (ed.), Des chevaux et des hommes. Équitation et société. Lausanne : Favre (« Caracole »)/Avignon : R.M.G.

1996. Champ de blé, champ de course. Nouveaux usages du cheval de trait en Europe. Paris : Jean-Michel Place.

Mennessier de la Lance, Général

1971. Essai de bibliographie hippique. Paris : Lucien Dorbon (1ère édition, Nieuwkoop : B. De Graaf 1915).

Milliet, Jacqueline \& et Jean-Pierre Digard

1989. « Une enquête sur les nouvelles sensibilités à l'égard des animaux domestiques ",

Anthropozoologica 10 : 31-47.

Picon, François-René

1999. « Le cheval dans le Nouveau Monde. Histoires culturelles », Études Rurales 151-152 : 51-75.

Pilley-Mirande, Natalie

2000. Le Secret des chuchoteurs américains. Paris : Zulma.

Rarey, John S.

1996. L'Art de dompter les chevaux. Préface de François-Paul de Guaita, présentation de Jean-Louis Gouraud. Lausanne : Favre (1ère édition 1858).

Raspail, Jean

1975. Journal peau-rouge. Paris : Laffont.

Roberts, Monty

1997. L'Homme qui sait parler à l'oreille des chevaux. Histoire de ma vie. Paris : Albin Michel.

Saurel, Étienne

1990. Histoire de l'équitation, des origines à nos jours. Paris : C. de Bartillat.

Serguine, Jacques

1985. Je suis de la nation du loup. Paris : Belfond.

Tourre-Malen, Catherine

2003. « Les à-côtés de l'équitation. Rapport à l'animal et pratique sportive », Études Rurales 165-166: 133-146.

s/presse. « Des amazones aux cavalières : avatar d'un loisir féminin », in D. Roche (ed.), Le Cheval et les loisirs (XVIe-XXe siècle). Paris : Association pour l'académie d'art équestre de Versailles.

Vergnaud, A.-D.

1860. Nouveau manuel complet d'équitation à l'usage des deux sexes. Paris : Manuels Roret.

\section{NOTES}

1. Ce travail s'inscrit dans le cadre d'une convention de recherche CNRS-Haras nationaux sur « Cultures équestres en crise : professionnels et usagers du cheval face au changement ", en partenariat avec la Commission européenne, Fonds social européen. 2. Cheval Magazine, n² 299, 1996, p. 55-57, et le numéro « spécial 25 ans », 1996, p. 55.

3. Procédé de dressage qui consiste à faire soulever par des aides la barre au moment où le cheval la franchit pour le forcer à lever davantage les pieds. 
4. Méthode de reproduction consistant à faire saillir une jument par un étalon sélectionné pour ses aptitudes par l'éleveur, les deux animaux étant présentés l'un à l'autre tenus à la main, à la différence de la monte dite " en liberté ».

5. Cette opinion, émise il y a une quinzaine d'années par l'éthologue américaine Marthe Kiley-Worthington (1989), a été récemment reprise avec éclat par la revue Cheval Loisirs (n 129, février 2003, pp. 68-70).

6. Présentation synthétique de ces méthodes dans Pilley-Mirande (2000).

7. Communiqué paru dans L'Éperon, $n^{\circ} 224$, juin 2003, p. 6.

8. Le lecteur que les ouvrages des « chuchoteurs » rebuteraient pourra avantageusement se limiter à la synthèse de Pilley-Mirande (2000).

9. Cheval Loisirs, $n^{\circ} 129$, février 2003, p. 37 (mes italiques).

10. Les Utes et les Apaches des Plaines voyaient dans les chevaux des "chiens sacrés ", ce qu'un auteur d'ethno-roman a judicieusement traduit par « surchiens » (Serguine 1985) -comment ne pas penser, ici, au processus de « canisation » du cheval auquel participe l'« équitation éthologique » (voir ci-dessus)?

11. Voir à cet égard les points de vue complémentaires de Digard (1992) et de Picon (1999).

12. Rarey 1858 : VIII; voir aussi Vergnaud $1860: 289$ et Mennessier de la Lance 1915, vol. II : 377 .

\section{RÉSUMÉS}

Le récent passage du cheval de la sphère de l'utilitaire à la sphère des loisirs a entraîné de profonds changements dans la population des cavaliers, dans les pratiques équestres et dans le statut culturel de cet animal. L'engouement actuel pour l'équitation dite « éthologique » s'inscrit dans ce mouvement : profitant à la fois d'une aspiration à d'autres rapports avec les animaux et d'une fascination pour une certaine image des Indiens d'Amérique du Nord, il participe à la « canisation » du cheval et au glissement de son statut vers celui d'animal de compagnie.

From riding schools to tipis. Ethological horse riding and Amerindians myths.

Recently, horses have left the utilitarian sphere to enter the leisure sphere. This has greatly changed the horsemen population, the way of riding and the cultural status of horses. The current feeling of interest for ethological equitation is part of this evolution, which is the result of both a wish to build new relations to animals and a fascination for Amerindians. These evolution contributes to the « doggization » of the horse, which progressively is becoming a pet.

De los picaderos a los tipis. « Equitación ecológica » y mitos indios.

El paso reciente del caballo de la esfera de lo utilitario hacia la esfera de las distracciones ha provocado cambios profundos en el conjunto de los jinetes, en las prácticas ecuestres y en el estatus de este animal. Dicho proceso involucra la afición actual a la equitación llamada " etológica » : contribuye en la «canisación » del caballo y en el desplazamiento de su estatus hacia el estado de animal de compañía, aprovechándose a la vez de un deseo de nuevas relaciones con los animales y de la fascinación para cierta imagen de los Indios de América del Norte. 
INDEX

Mots-clés : cheval, domestication animale, équitation, éthologie, France, Indiens d'Amérique du Nord

Keywords : Amerindians, animals' domestication, equitation, ethology, horse

\section{AUTEUR}

JEAN-PIERRE DIGARD

Centre de Recherche Pluridisciplinaire d'Ivry, CNRS, 27 rue Paul Bert, 94204 Ivry-sur-Seine Cedex 